\title{
Impact of Circular Field in Underwater Wireless Sensor Networks
}

\author{
Syed Agha Hassnain Mohsan ${ }^{1}$, Mushtaq Ali Khan², Arfan Mahmood ${ }^{3}$, Muhammad Hammad Akhtar ${ }^{4}$ \\ Hussain Amjad ${ }^{5}$, Asad Islam ${ }^{6}$, Alireza Mazinani ${ }^{7}$, Syed Muhammad Tayyab Shah ${ }^{8}$ \\ Department of Electrical Engineering, COMSATS University Islamabad (CUI), Pakistan ${ }^{1,8}$ \\ Ocean College, Zhejiang University, Zhoushan, China, ${ }^{2,5}$ \\ Complex Networks and Control Lab, bhanghai Jiao Tong University, Shanghai, China ${ }^{3}$ \\ National University of Science and Technology (NUST), Islamabad, Pakistan ${ }^{4}$ \\ Beihang University (BUAA), Beijing China ${ }^{6,7}$
}

\begin{abstract}
Underwater Wireless Sensor Networks (UWSNs) face challenges regarding high propagation delay, limited bandwidth, 3D topology and excessive energy consumptions. In this paper, a routing scheme with circular field is proposed for an efficient collection of data packets by using two mobile sinks in UWSNs. Results of proposed scheme are compared with previous implemented schemes which are used to measure the usage of mobile sink in the collection of data packets. In this study, we have compared the proposed scheme with current state-of-the-art routing protocols. The statistical significance of this work was analyzed in MATLAB. Marked observations to emerge from obtained results include an improvement in lifetime, increased throughput, increment in alive nodes and balanced energy consumption. In our view, these results strengthen the validity of proposed circular field. A significant increase in received packets is observed because maximum nodes are alive till 1500 rounds which provide maximum communication and less chance of creating void holes.
\end{abstract}

Keywords-Component; underwater wireless sensor networks; mobile sink; routing scheme; circlular field

\section{INTRODUCTION}

A considerable research has been carried out on terrestrial sensor networks in different aspects but currently research community is attracted towards a new era of research in Underwater Wireless Sensor Networks (UWSNs). Human beings are unable to work at high pressure ocean environment for a long time. On the other hand, Terrestrial Wireless Sensor Networks (TWSNs) are feasible but it cannot be considerable for UWSNs because signals transmission is elusive due to higher attenuation of aquatic environment. Underwater Wireless Sensor Network (UWSN) consists of various components such as sensors and vehicles deployed in acoustic area for data collection and collaborative monitoring tasks. Generally, UWSNs differ from TWSNs in terms of sensors cost, dense deployment of these sensors, communication method and maximum power required for this communication. USWNs are being used in several applications of underwater environment such as observation of chemical pollution, seismic activities, submarine movements, monitoring of marine creatures, distributed tactical surveillance, disaster prevention and military applications. However, UWSNs face challenges regarding narrow bandwidth, 3D topology, long propagation delay, media access control, attenuation, harsh geographical atmosphere, power constraints, losses of connectivity and high bit error rates. Though, research community has provided several techniques to cope with these challenges but still gap lies for research because of variable characteristics of ocean. Usually underwater communication is different from air due to varying conductivity, permittivity and permeability [1-2] parameters.

This technology has already achieved a great stature among researchers. With the progress in underwater technologies, a growing research inclination has been noticed by industry as well as academia in UWSNs [3-4]. Currently, acoustic signals are in great consideration for underwater applications as they are more feasible for underwater communications with low absorption rate [5]. Long transmission range can be achieved by acoustic communication. However, it faces time-varying signal distortion and transmission losses. In UWSNs, reliability and stability of network has paramount importance. The maintenance of network lifetime in UWSNs is big issue as it's difficult to replace those batteries which provide power to sensor nodes. Recently, researchers are working in underwater wireless power transfer to tackle these challenges. In addition, TWSNs routing protocols cannot be implemented to UWSNs because of limited bandwidth, long propagation delay, energy constraints, high mobility and non-uniform 3D topology. Researchers have suggested several routing protocols for UWSNs. Some routing protocols can enhance the stability duration of network on the basis of throughput while others increase throughput on a compromise over packet delay [5]. In these protocols clustering is considered as the pragmatic method for load balancing in UWSNs because sensor nodes are categorized in different groups. Among these sensor nodes, cluster head is used as leading head [6]. Some researchers proved that mobile sink is an effective way to enhance throughput [7]. In order to collect data, these mobile sinks can connect to individual sensor node or full cluster. In a research study discussed in [8], authors utilized two mobile sinks to gather data from sparse regions. It is noticed when both sinks are located at adjacent positions [9] then throughput is affected due to same sensor nodes.

Considering above discussion, we were inspired and implemented a routing protocol which uses two mobile sinks without covering same transmission area. The overriding objective of this study is to seek an intelligent use of mobile

\footnotetext{
*Corresponding Author
} 
sinks. A previous routing scheme Towards Efficient Sink Mobility (TESM) [10] is implemented with a circular field while previously it was taken as rectangular field. A. Yahya et al. discussed that high MUR represents the effective use of mobile sinks and obtained MUR results of this work are compared with SEEC and TESM. The paper is organized as: Section 2 is based on relevant research discussion. Section 3 will focus on motivation behind this present study. The impacts of circular network field are evaluated in Section 4 while results discussion has been carried out in Section 5. Conclusion and future work is presented in last sections.

\section{RELEVANT RESEARCH WORK}

Since many years' researchers have been focusing to design several UWSNs protocols for qualitative and effective research analysis. Research community is working with the aim of paving a way for new era of underwater actuation and monitoring applications. UWSNs will help us to know ocean with its envisioned landscape of applications. Researchers have suggested various routing schemes to achieve better results as Goyal et al. [11] highlighted capabilities and limitations on the basis of a survey on UWSN with data aggregation. Khan et al. [7] designed a routing scheme named as weighting depth and forwarding area division depth base routing protocol (WDFAD-DBR). They achieved reliable results in sparse deployment by minimizing void holes issue. They proposed balanced energy consumption on the basis of division criteria of forwarding regions. A SEEC protocol is proposed in [9]. F. Pan et al. [12] presented an energy-balanced algorithm based on energy level partition and depth threshold. In this protocol, mobile sinks can collect data at sparse region and they implied clustering on dense regions. Wan et al. [13] suggested an energy efficient scheme based on multilevel cluster selection criteria which aims to choose cluster head according to high residual energy and less radius from sink. In [14], network lifetime of UWSNs is maximized by the assistance of sink mobility with geographic routing protocol. A reliable energy efficient routing protocol based on clustering is simulated [15] for UWSNs. Authors proposed that clustering technique is useful to reduce energy resources consumption for a sensor network. Researchers have proposed a routing protocol AEDG which involves effective data collection through AUV [16]. The usage of multiple mobile sinks to enhance network lifetime is discussed in previous studies. Authors validated that a better network lifetime can be achieved through assigning a predefined path to mobile sinks for movement. While research study in [17] focuses on relation between ocean currents and energy hole problems. This study introduces drift speed calculations. It mentions that drift speed can be calculated at any time if the current location of sensor nodes is known. Mobile sink changes its speed after sensing the ocean current accordingly. It is worth noting that throughput of this proposed scheme is also better. Many researchers have presented current research challenges of intrinsic properties, topology control and potentials in UWSNs through several topology control algorithms. M. Ma et al. [18] have used a hybrid approach to maximize network lifetime and minimize the energy holes. In [19], authors proposed a mechanism for data collection involving large scale multihop sensor networks. In a recent work, authors designed data uploading decision making on the basis of similar data collected [20]. In this research study, authors maximized the network lifetime in AEDG as AUV is sent to gather data from gateway nodes. Wang et al. deployed a selection algorithm based on Heuristic Adaptive Sink Sensor for prolonging network lifetime and energy saving purposes in this study. In another research study discussed in [11], Hop ID is given to specific sensor nodes according to hope count from sink. Habib M. Ammari [20] investigated sink mobility in a circular field. The field consists of concentric circular bands to find a possible solution for optimal sink mobility. In a Doctoral research study, Erik F. Golen [21] briefly discussed sensing and communication coverage of sensors in circular regions. In a recent research work, Chaima Zidi [22] illustrated energy efficient UASNs in which sensors were deployed in a circular sensor field. In a systematic review [23], modulation techniques and channel coding is briefly discussed for discrete underwater sensor nodes.

\section{Motivation}

As discussed in [10], authors introduced a novel routing scheme TESM for efficient sink utility in which two mobile sinks are involved to gather required data. Authors introduced a novel metric Mobile Sink Utility Ratio (MUR) which is useful to measure the utility of mobile sinks while data gathering. They have used a rectangular field in their proposed scheme while current study focuses on circular field. Results have been compared with routing scheme for efficient sink utility and other previously implemented routing schemes with regard to network lifetime, throughput, residual energy, stability and instability period.

\section{SCHEME WITH CIRCUlAR FIELD}

An efficient use of mobile sinks in UWSNs appears as maximum throughput with minimum energy consumption. In this study, a routing scheme TESM discussed in [10] is considered with a different circular field. The achieved results will be discussed in next sections.

\section{A. Network Model}

This study includes a network circular field with a logical division of two equal parts. Circular field has a diameter of 100 meters. This logical division is adopted for an efficient movement of mobile sinks. It is worth noting, three sinks are used: one is static while other two are dynamic. Two mobile sinks are placed in each divided portion of circular field to gather data. The static sink is located above central position of circular network field. Network model can be seen in Fig. 1.

\section{B. Energy Consumption Model}

The transmission distance is represented as D and B represents the total size of packet. $\boldsymbol{B}_{\boldsymbol{d}}$ and $\boldsymbol{R}_{\text {disp }}$ show bit duration and radio dissipation respectively. The total energy required [10] for sending $\boldsymbol{B}$ bits packets at transmission distance $\boldsymbol{D}$ can be calculated by following equation:

$$
\boldsymbol{E}_{\text {trans }}(\boldsymbol{B}, \boldsymbol{D})=\left(\boldsymbol{B} * \boldsymbol{R}_{\text {disp }}\right)+\left(\boldsymbol{B} * \boldsymbol{B}_{\boldsymbol{d}}\right)
$$

While the energy consumed during packet reception [11] can be calculated by using equation 2 .

$$
\boldsymbol{E}_{\text {rcv }}(\boldsymbol{B}, \boldsymbol{D})=\boldsymbol{B} * \boldsymbol{R}_{\text {disp }}
$$




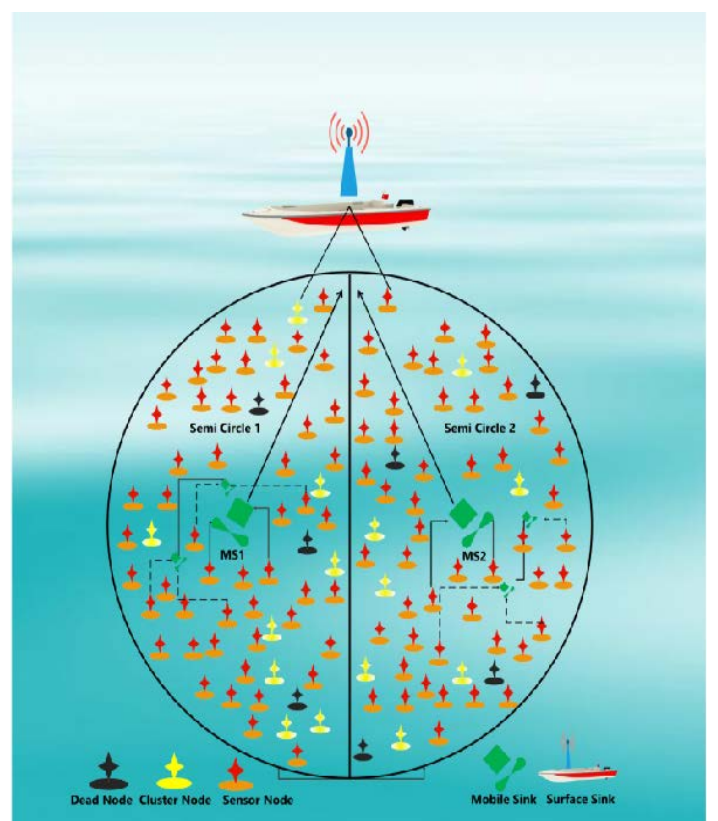

Fig. 1. Network Model.

The residual energy Eris for each sensor node is obtained from equation 3.

$\boldsymbol{E}_{\text {ris }}=\boldsymbol{E}_{\text {int }}-\left(\boldsymbol{E}_{\text {rcv }}+\boldsymbol{E}_{\text {trans }}\right)$

Here $\boldsymbol{E}$ int shows the initial energy level of any sensor node.

\section{Circular Network Field Configuration}

Here, the procedure provided in literature [10] is followed to implement routing scheme with a circular network field. For this purposed work, the circular field is divided into two equal regions. Two mobile sinks MS1 and MS2 are deployed at center point of each region at same distance from center of circle. Nodes are randomly deployed in circular field. MS1 is present in semi-circle 1 while MS2 is present in semi-circle 2. Both sinks move at an angle of $45^{\circ}$ directing upward towards base station deployed at terrestrial region. Corresponding sink receives data from nodes when sink appears within transmission range of these nodes. Transmission range and some others parameters are defined in next section. It is required to find sparse and dense areas after network deployment. Cluster is formed where nodes are dense. Random selection of cluster head is made according to highest energy. It is worth noting; in every round each member is provided opportunity to become cluster head. Cluster head can communicate to mobile sink and base station. Remaining nodes without cluster can communicate each other and can send packets to mobile sink as well. The coordinates of mobile sinks can be found from below equations.

$X=r_{i} \cos \theta_{i}$

$Y=r_{i} \sin \theta_{i}$

The circular network field is divided into two semi-circle regions where radius of each region is $r=50 \mathrm{~m}$. The origin of circle is represented as $O(x, y)$.

$\boldsymbol{O}(\boldsymbol{x}, \boldsymbol{y})=(\mathbf{0}, \mathbf{0})$
While considering radius $r=50 \mathrm{~m}$, the area of two semicircles can be calculated by following equation 7 .

$A_{i}=\frac{\pi r^{2}}{2}$

$i=1,2$ for two sub regions. As area calculation for both sub-regions is expressed in above equation, the total area of the whole circular network field is calculated. Equation 8 expresses the division criteria for each sector of circular network field.

$S_{n}=\int_{0}^{\pi}\left(\frac{\theta}{360}\right) * \pi * r^{2}$

Both mobile sinks as represented with MS1 and MS2. Mobile sinks move $45^{\circ}$ in upward direction in a tilt way towards base station.

$M S 1=\sum_{t=0}^{n}\left(\theta+45^{\circ} / 360\right) * \pi * r_{i}^{2}$

Similarly

$M S 2=\sum_{t=0}^{n}\left(\theta+45^{\circ} / 360\right) * \pi * r_{i}^{2}$

Here $n$ depicts total sectors while $t=0$ represents that MS1 and MS2 are at center position of first and second sub-region.

\section{RESUlTS DisCUSSION}

To evaluate performance, the results are compared with previously implemented routing protocols SEEC, TESM and DBR. Simulation work was carried out by designing a complete UWSN environment through MATALB [11]. Table I summarizes simulation parameters.

To evaluate performance of routing protocols, proposed study considers primary metrics of network stability and instability period, packets received per round, network residual energy, network throughput, mobile sink utility ratio and packets received at sink.

\section{A. Network Lifetime and Stability Period}

By considering circular field, network lifetime is improved as it can be seen in Fig. 2. SEEC did not use two mobile sinks efficiently as their movement is in sparse regions only. Considering this transmission range, it is not suggested to use mobile sinks based on sparse area. Both MS1 and MS2 are restricted in each semi-circle. Both mobile sinks are moved upward in a tilt position towards base station. Due to this movement, a greater stability period performance is obtained as compared to SEEC, DBR and TESM. The restriction on movements of both mobile sinks in each semi-circle increases the stability period. It can be clearly seen that network lifetime results of proposed work are better comparatively with previous schemes. Fig. 2 validates more nodes are alive in this work than SEEC, DBR and TESM.

\section{B. Network Residual Energy}

In SEEC, clustering is achieved in top four dense regions while using only two mobile sinks in sparse regions. In DBR, energy consumption is more due to selection criteria because forward nodes are selected on lower depths only. In obtained results, both mobile sinks are restricted in each potion of circular field. If number of dense regions exceeds highly than sparse regions, mobile sinks movement will increase energy 
consumption only. The distance between nodes is larger in sparse regions whereas it is smaller in case of dense regions. Residual energy of this proposed work compared with SEEC, TESM, DBR is plotted in Fig. 3.

TABLE I. SIMULATION PARAMETERS

\begin{tabular}{|l|l|}
\hline Parameters & Values \\
\hline Data rate & $16 \mathrm{Kbps}$ \\
\hline Number of nodes & 100 \\
\hline Packet size & $50 \mathrm{Bytes}$ \\
\hline Center frequency & $30 \mathrm{KHz}$ \\
\hline Initial energy of nodes & $5 \mathrm{~J}$ \\
\hline Receive power of packet & $0.1 \mathrm{~W}$ \\
\hline Transmission power of packet & $2 \mathrm{~W}$ \\
\hline Running rounds & 3500 \\
\hline No. of Sinks & 2 \\
\hline Radius of Circle & $50 \mathrm{~m}$ \\
\hline Transmission range & $50 \mathrm{~m}$ \\
\hline
\end{tabular}

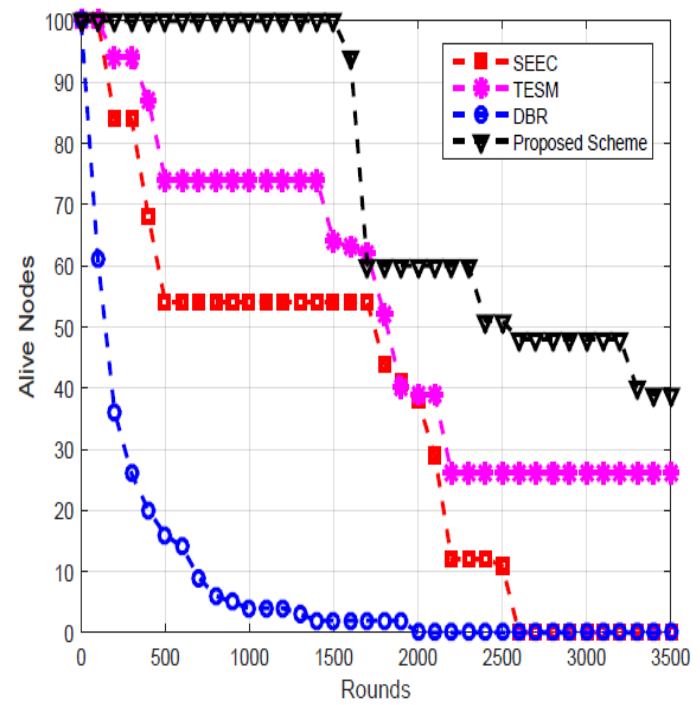

Fig. 2. Network Lifetime.

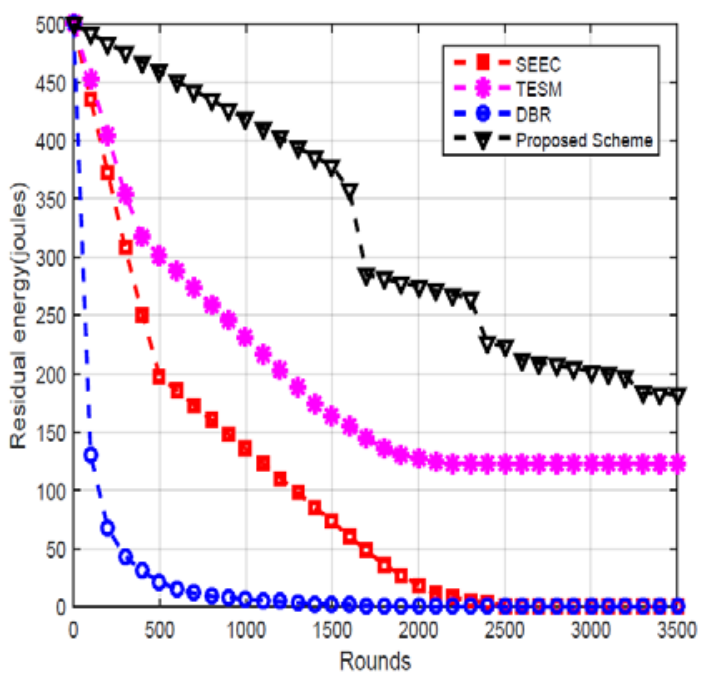

Fig. 3. Residual Energy.

\section{Network Throughput}

In Fig. 4, the received packets per round at any sink are more than SEEC, TESM and DBR. An increased throughput in this proposed work with circular field can be noticed as sensors are omni-directional as well. In SEEC, mobile sinks movement is according to sparse region and in TESM it is based on selection criteria opted for forwarding nodes.

Fig. 5 is showing the overall received packets at sink. Therefore, it gives the addition of received packets in both previous round and next round. Graph shows that more packets are received at sink than SEEC, TESM and DBR.

\section{Mobile Sink Utility Ratio}

Fig. 6 is showing the usage of mobile sink is high for data packets collection as compared to SEEC and lower than TESM.

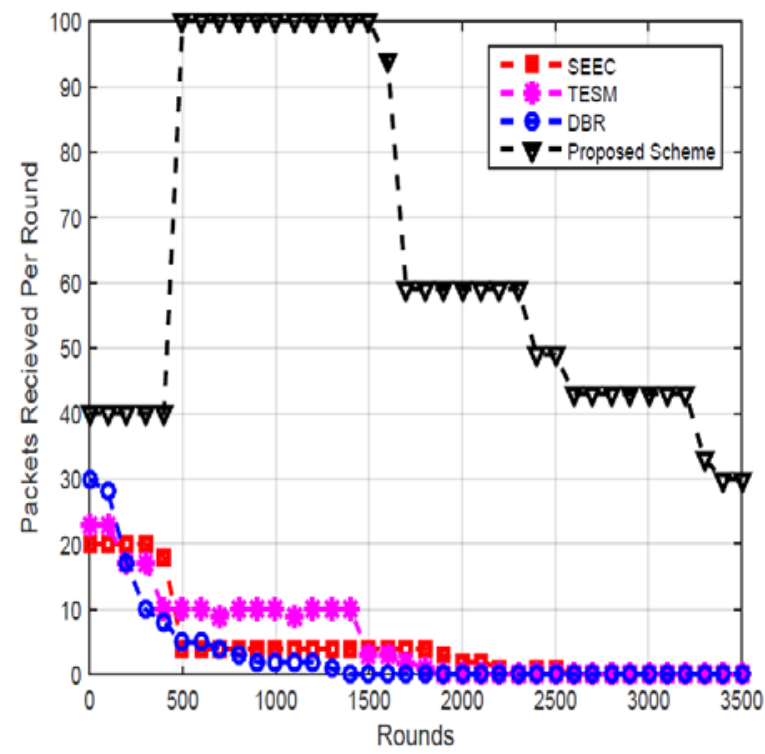

Fig. 4. Packets Received Per round.

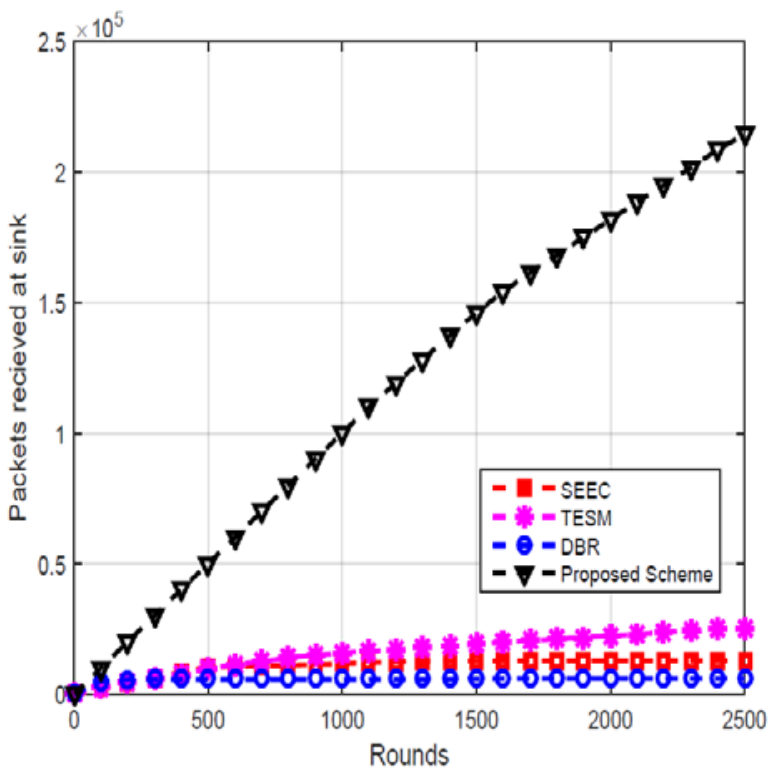

Fig. 5. Packets Received at Sink. 


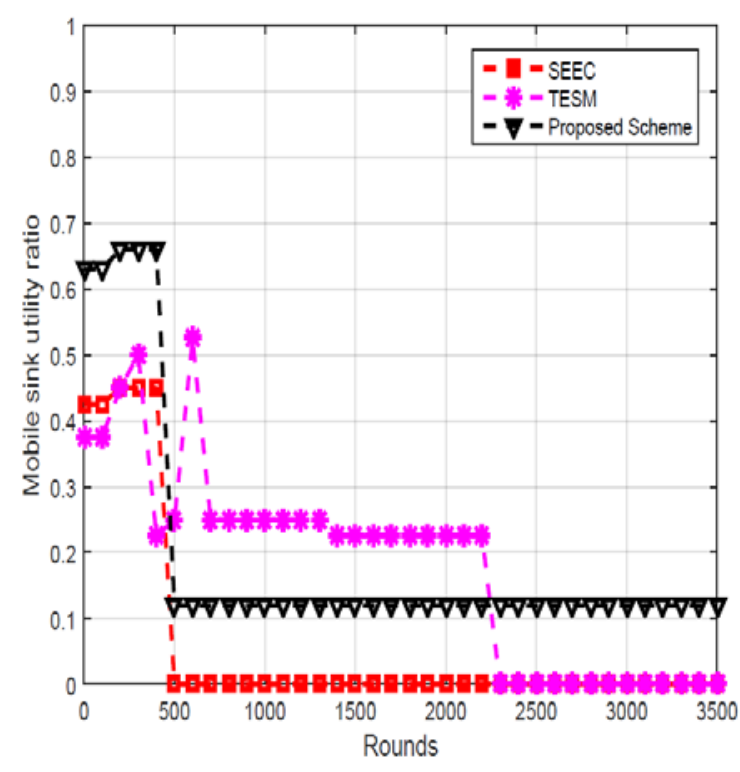

Fig. 6. Packets Received at Sink.

Results of this scheme are not compared with other routing schemes as only SEEC and TESM use mobile sinks among all schemes. Therefore, proposed work is compared with SEEC and TESM to properly analyze the efficient utilization of mobile sinks. In these results, motion pattern of mobile sinks gives high MUR than SEEC but less than TESM because mobility pattern performance of TESM is better. In this case, high MUR results as high throughput while it is noticed that the overall packets collected in SEEC by mobile sinks are lower which results as low MUR.

\section{E. Dead Nodes}

Herein, stability period of implemented routing scheme TESM [8] with a circular field is shown. A good performance is noted in terms of stability period as compared to previous scheme of SEEC, DBR and TESM. In this work, network remains stabilize for a long time with a minimal consumption of energy. In proposed study, dead nodes are less than previous scheme as it is presented in Fig. 7.

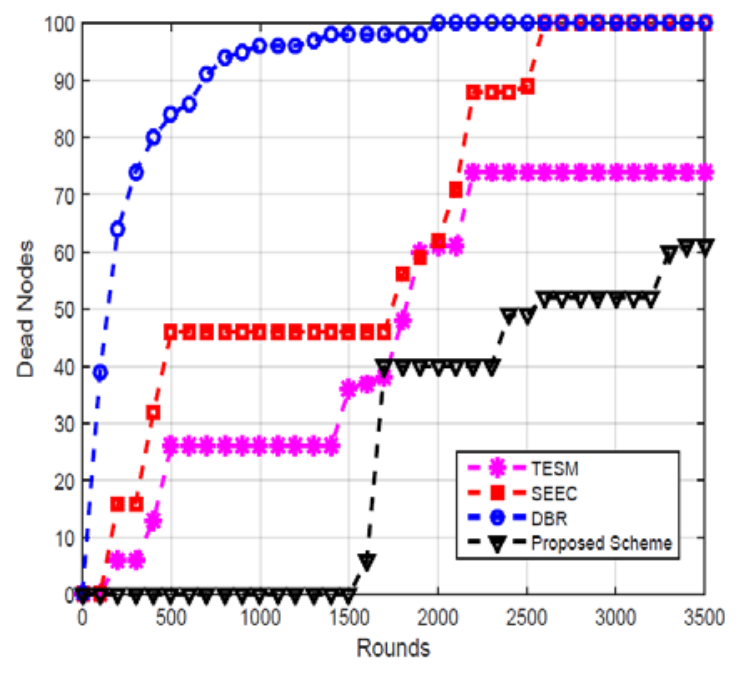

Fig. 7. Stability and instability perdiod of network

\section{Vi. Performance Tradeoff}

The proposed work gives better network lifetime, throughput and balanced energy consumption. It is worth noting that more nodes are alive while dead nodes are less. Thus, proposed work concludes a considerable progress of this work in aspects of stability period, residual energy and throughput.

\section{VII.CONCLUSION}

Results presented in this work illustrate that optimum and efficient use of mobile sinks is crucially important in enhancing network lifetime and throughput. This work has led us to conclude that a proper distribution of network field and shape is pivotal. Therefore, proposed work is compared with SEEC and TESM to properly analyze the efficient utilization of mobile sinks. In addition, the results of any routing scheme can be improved if proper use of resources in the protocol is maintained. In this study, the results while considering a circular field are compared with previous implemented schemes. To sum up our work, the proposed circular field is better in throughput, network lifetime and balanced energy consumption. A significant increase in received packets is observed because maximum nodes are alive till 1500 rounds which provide maximum communication and less chance of creating void holes. Thus, a good performance is noted in terms of stability period as compared to previous scheme of SEEC, DBR and TESM.

\section{FUTURE WORK}

In future, we are aiming to consider scarcity control in order to improve network lifetime. Though proposed work is energy efficient and throughput is also achieved but it will cause delay. Current work includes an investigation of possible techniques to reduce this delay. The delay in each round will be evaluated in future research work. Future work is to concentrate on minimizing interference in dense area which will make reliable delivery of data packets in any network. Holding time phenomenon towards reducing packet drop ratio is deferred to future work.

\section{REFERENCES}

[1] Hassnain, S. A., M. J. Mughal, and Q. A. Naqvi. "Layered Chiral Spheres with Zero Backscattering." 2019 Photonics \& Electromagnetics Research Symposium-Fall (PIERS-Fall). IEEE, 2019.

[2] Hassnain, S. A., M. J. Mughal, and Q. A. Naqvi. "Analysis of Effective Medium Parameters on Polarizability of Homogeneous Chiral Sphere." 2019 Photonics \& Electromagnetics Research Symposium-Fall (PIERSFall). IEEE, 2019.

[3] Li, Ning, et al. "A probabilistic and highly efficient topology control algorithm for underwater cooperating AUV networks." Sensors 17.5 (2017): 1022.

[4] Li, Ning, et al. "A survey on underwater acoustic sensor network routing protocols." Sensors 16.3 (2016): 414.

[5] Yan, H.; Shi, Z.J.; Cui, J.H. DBR: Depth-based routing for underwater sensor networks. In International Conference on Research in Networking; Springer: Berlin/Heidelberg, Germany, 2008; pp. 72-86.

[6] Han, G.; Jiang, J.; Bao, N.; Wan, L.; Guizani, M. Routing protocols for underwater wireless sensor networks. IEEE Commun. Mag. 2015, 53, 72-78.

[7] Khan, Jawaad Ullah, and Ho-Shin Cho. "A distributed data-gathering protocol using AUV in underwater sensor networks." Sensors 15.8 (2015): 19331-19350. 
[8] Coutinho, Rodolfo WL, et al. "Underwater wireless sensor networks: A new challenge for topology control-based systems." ACM Computing Surveys (CSUR) 51.1 (2018): 1-36.

[9] Sher, A.; Javaid, N.; Azam, I.; Ahmad, H.; Abdul, W.; Ghouzali, S.; Niaz, I.A.; Khan, F.A. Monitoring square and circular fields with sensors using energy-efficient cluster-based routing for underwater wireless sensor networks. Int. J. Distrib. Sens. Netw. 2017, 13, 1550147717717189.

[10] Yahya, Aqeb, et al. "Towards efficient sink mobility in underwater wireless sensor networks." Energies 11.6 (2018): 1471.

[11] Goyal, Nitin, Mayank Dave, and Anil K. Verma. "Data aggregation in underwater wireless sensor network: Recent approaches and issues." Journal of King Saud University-Computer and Information Sciences 31.3 (2019): 275-286.

[12] Feng, Pan, et al. "Improved energy-balanced algorithm for underwater wireless sensor network based on depth threshold and energy level partition." EURASIP Journal on Wireless Communications and Networking 2019.1 (2019): 1-15.

[13] Wan, Zhiping, et al. "An energy-efficient multi-level adaptive clustering routing algorithm for underwater wireless sensor networks." Cluster Computing 22.6 (2019): 14651-14660.

[14] Ahmed, Farwa, et al. "Mobile sinks assisted geographic and opportunistic routing based interference avoidance for underwater wireless sensor network." Sensors 18.4 (2018): 1062.
[15] Huang, Xiangdang, Shijie Sun, and Qiuling Yang. "Data Uploading Strategy for Underwater Wireless Sensor Networks." Sensors 19.23 (2019): 5265.

[16] Khan, Jawaad Ullah, and Ho-Shin Cho. "A distributed data-gathering protocol using AUV in underwater sensor networks." Sensors 15.8 (2015): 19331-19350.

[17] Chen, Y.S.; Lin, Y.W.Mobicast routing protocol for underwater sensor networks. IEEE Sens. J. 2013, 13, 737-749.

[18] Ma, Ming, and Yuanyuan Yang. "SenCar: An energy-efficient data gathering mechanism for large-scale multihop sensor networks." IEEE Transactions on Parallel and Distributed Systems 18.10 (2007): 14761488.

[19] Wang, Xingwang, et al. "Has4: A heuristic adaptive sink sensor set selection for underwater auv-aid data gathering algorithm." Sensors 18.12 (2018): 4110.

[20] Ammari, Habib M. "A unified framework for k-coverage and data collection in heterogeneous wireless sensor networks." Journal of Parallel and Distributed Computing 89 (2016): 37-49.

[21] Golen, Erik. "Intelligent deployment strategies for passive underwater sensor networks." (2009).

[22] Zidi, Chaima. Energy efficient underwater acoustic sensor networks. Diss. Université Sorbonne Paris Cité, 2018.

[23] Mehedi, Syed Agha Hassnain Mohsan Md, et al. "A Systematic Review on Practical Considerations, Recent Advances and Research Challenges in Underwater Optical Wireless Communication." IJACSA 11-7 (2020). 